\title{
Effect of amlodipine besylate combined with acupoint application of traditional Chinese medicine nursing on the treatment of renal failure and hypertension by the PI3K/AKT pathway
}

\author{
LIPING FENG $^{1 *}$, JIAHANG SU$^{2}$, RONGXIANG CHI ${ }^{3 *}$, QIAO ZHU ${ }^{4}$, SHUGUANG LV $^{4}$ and WENJING LIANG \\ ${ }^{1}$ Department of Rehabilitation, ${ }^{2}$ Clinical Pharmacy Room, Departments of ${ }^{3}$ Nursing, ${ }^{4}$ Cardiology and ${ }^{5}$ Public Health, \\ Yantai Hospital of Tradition Chinese Medicine, Yantai, Shandong 264000, P.R. China
}

Received September 16, 2018; Accepted February 8, 2019

DOI: $10.3892 /$ ijmm.2019.4104

\begin{abstract}
To study the effect and molecular mechanisms of amlodipine besylate combined with acupoint application of traditional Chinese medicine nursing on the treatment methods of renal failure and hypertension. A total of 60 cases of renal failure hypertension were randomly divided into the Control group and the Treatment group. The control group was treated with amlodipine besylate, while the treatment group was treated with amlodipine besylate combined with acupoint application of traditional Chinese medicine nursing. A rat model of renal failure hypertension was established. Rats were divided into the sham group, model group, NC group (treated with amlodipine besylate) and treatment group (treated with amlodipine besylate combined with acupoint application of traditional Chinese medicine nursing). Rats were given drugs at 10-20 weeks of age to observe their general condition and detect changes of blood pressure, blood biochemical indices and urine index. The pathological changes of renal tissue
\end{abstract}

Correspondence to: Dr Wenjing Liang, Department of Public Health, Yantai Hospital of Tradition Chinese Medicine, 39 Xingfu Road, Zhifu, Yantai, Shandong 264000, P.R. China

E-mail: ggws2127338@163.com

Dr Shuguang Lv, Department of Cardiology, Yantai Hospital of Tradition Chinese Medicine, 39 Xingfu Road, Zhifu, Yantai, Shandong 264000, P.R. China

E-mail: lvdocsci@yeah.net

${ }^{*}$ Contributed equally

Abbreviations: PI3K, phosphoinositide 3-kinase; AKT, protein kinase B; VEGF, vascular endothelial growth factor; MMP9, matrix metallopeptidase 9; ET-1, endothelin-1; NF-кB p65, nuclear factor- $\kappa \mathrm{B}$ p65; BUN, blood urea nitrogen; CRE, carbapenem-resistant Enterobacteriaceae; NAG, N-acetyl- $\beta$-D-glucosaminidase; Ccr, creatinine clearance; TP, total protein; UP, urine protein

Key words: amlodipine besylate, acupoint application, renal failure, hypertension; phosphoinositide 3-kinase, protein kinase B pathway were examined by hematoxylin and eosin staining, and the expression of vascular endothelial growth factor (VEGF) and matrix metalloproteinase (MMP)9 were detected by immunohistochemistry. Reverse transcription-quantitative polymerase chain reaction was used to determine mRNA expression of phosphoinositide 3-kinase (PI3K), protein kinase B (AKT) and endothelin (ET)-1 and western blotting was used to detect the expression of phosphorylated (p)-PI3K/PI3K, p-AKT/AKT and p-nuclear factor (NF)- $\kappa \mathrm{B}$ p65/NF- $\mathrm{B}$ p65 protein. Systolic and diastolic blood pressures in Treated group patients were significantly lower compared with in Control group patients. The systolic and diastolic blood pressure of rats were significantly decreased and blood urea nitrogen (BUN), carbapenem-resistant Enterobacteriaceae (CRE), N-acetyl- $\beta$-D-glucosaminidase (NAG), urine protein (UP) and blood urea protein (BUP), contents were significantly decreased following amlodipine besylate treatment. The expression of VEGF and matrix metallopeptidase 9 protein were significantly decreased, but the expression of PI3K, AKT mRNA and p-PI3K/PI3K, p-AKT/AKT protein were significantly increased. ET-1 mRNA and p-NF- $\kappa$ B $\mathrm{p} 65 / \mathrm{NF}-\kappa \mathrm{B}$ protein were significantly increased. The pathological alterations of renal tissue were improved and the pathological changes of glomerulus, tubule and interstitium were alleviated. Amlodipine besylate combined with acupoint application of traditional Chinese medicine nursing can effectively reduce the systolic pressure and diastolic pressure of patients, and improve the symptoms and signs of patients, which may be associated with the regulation of the expression of PI3K/AKT pathway, so as to regulate the expression of BUN, CRE, UP, BUP and NAG.

\section{Introduction}

Renal failure is a pathological state of partial or complete loss of renal function. It can be divided into acute and chronic type disease (1). Acute renal failure is caused by a variety of diseases, resulting in the loss of excretion by the two kidneys over a short period of time (2). Chronic renal failure (CRF) is a combination of clinical symptoms formed by the development of chronic kidney disease, which is caused by various etiologies (3). Renal hypertension is mainly due to the increase 
of blood pressure caused by renal parenchymal diseases and renal artery diseases. It is also called renal hypertension in symptomatic hypertension (4).

Amlodipine besylate is a dihydropyridine calcium antagonist (5). The contraction of myocardium and smooth muscle depends on the extracellular calcium ions entering the cell through specific ion channels (6). Amlodipine besylate selectively inhibits calcium ion transmembrane movement into smooth muscle cells and cardiac muscle cells, and the effect on smooth muscle is greater compared with the effect on the myocardium (7). The interaction between calcium ions and its calcium channel depends on the gradual rate of binding and dissociation between the receptor and its calcium channel (8). Amlodipine besylate is a peripheral artery dilator, acting directly on vascular smooth muscle, reducing peripheral vascular resistance, thereby lowering blood pressure. Certain western antihypertensive drugs have a number of shortcomings and side effects, including a decreased libido, liver damage and metabolic abnormalities. Traditional Chinese medicine treatment has a number of advantages, including fewer side effects, improvement of symptoms, protection of target organs, reduction of complications, low price and good patient compliance. Therefore, the use of Chinese medicine combined with western medicine treatment has great advantages over the use of Western medicine alone $(9,10)$.

Acupoint application therapy is guided by the holistic view of traditional Chinese medicine. Acupoint application of traditional Chinese medicine nursing (AA-TCM nursing) is the use of the principle of transdermal absorption on the surface of the human body to relieve the pain. Acupoint sticking is widely used in the clinic. It is a safe and stable treatment. Acupoint sticking therapy is a non-invasive pain acupoint therapy based on the theory of meridians and collaterals of traditional Chinese medicine (11). For a long time, experts and scholars have conducted literature studies on the literature of past dynasties, and investigated the results. Acupoint therapy is not only used to treat common diseases, but also used to treat pulmonary tuberculosis, liver cirrhosis, coronary heart disease, hypertension, infectious diseases and other difficult diseases (12).

Serine/threonine kinase AKT (also known as protein kinase $\mathrm{B}$ or $\mathrm{PKB}$ ), as a proto oncogene has become a major concern in the medical community, because it serves an important role in regulating various cell functions, including metabolism, growth, proliferation, survival, transcription and protein synthesis (13). Factors that can activate the cascade amplification of AKT signals, including receptor tyrosine kinase, integrin, B cells and $\mathrm{T}$ cell receptors, cytokine receptors, $G$ protein coupled receptors, and other stimuli that can induce phosphatidylinositol by phosphatidylinositol 3 kinase (PI3K) induced by phosphatidyl inositol (PIP3) (14). These lipids can be used as a mooring site for the protein membrane of the pleckstrin homology domain, including AKT and its upstream activator, phosphoinositide dependent protein kinase-1 (PDK1). PDK1 located in the cell membrane phosphorylates threonine at the 308 site of AKT, resulting in partial activation of AKT. Serine 473 sites are phosphorylated by mammalian target of rapamycin complex 2 , which can stimulate the complete enzyme activity of AKT (15). PI3K associated kinase family members, including DNA-PK, can also phosphorylate AKT serine at 473 sites. In addition, tumor suppressor gene phosphatase and tensin protein homologue protein also inhibits AKT activity by dephosphorylation of PIP3 (16). Endothelin 1 (ET-1), also known as preproendothelin-1, is a potent vasoconstrictor that in humans is encoded by the EDN1 gene and produced by vascular endothelial cells (17). The protein encoded by this gene is proteolytically processed to release a secreted peptide termed ET-1 (18).

In the present study, experiments were designed to investigate the effect and molecular mechanisms of amlodipine besylate combined with AA-TCM nursing on the treatment of renal failure and hypertension via the PI3K/AKT pathway.

\section{Materials and methods}

Patients and methods. The study was approved by the Yantai Traditional Chinese Medicine Hospital (Yantai, China). Informed consent was obtained from all participants. All included patients fulfilled criteria and completed the study.

Characteristic of studied groups. A total of 60 hypertensive patients with renal failure from the Department of Cardiology outpatient and hospitalization in the Yantai traditional Chinese Medicine Hospital were included in the study. There were 26 males and 34 females with an average age of 61 years. They were randomly divided into two groups, 30 cases in each group, group Control: Average age (60.80 \pm 13.45 years), average duration of treatment $(6.43 \pm 7.11$ years $)$ and male and female ratio $(15 / 15)$; Treatment group: Average age $(61.83 \pm 13.03$ years), average duration $(9.06 \pm 9.06$ years), and male and female ratio (11/19). The diagnostic scale was formulated according to the guideline 2010 for hypertension prevention and treatment in China (19). Diagnostic criteria for adult hypertension: i) Systolic pressure is $>140 \mathrm{mmHg}$ and (or) diastolic pressure $>90 \mathrm{mmHg}$; ii) serum creatinine $<364 \mu \mathrm{mol} / \mathrm{l}$; and iii) different levels of digestive tract symptoms and mild anemia. There was no significant difference in the duration, age, sex and grade of hypertension between the two groups. Causes of renal failure and subsequent treatment are listed as follows: i) Renal failure and hypertension patients over 18 years old; ii) it is in line with grade 1 and 2 hypertension, but no serious cardio pulmonary disease; iii) those taking blood pressure drugs who did not control blood pressure maintained the same dose and dosage during treatment, while those who did not take antihypertensive drugs did not take antihypertensive drugs during treatment; and iv) the patient's informed consent was good, and the compliance was good, respectively. Subjects were excluded from the study if they met these criteria: i) Patients with secondary hypertension; ii) pregnant and lactation women; and iii) poor consent and poor compliance.

Blood pressure measurement. The diagnosis of hypertension is based on the diagnostic criteria of hypertension in the guiding principles of new drugs: i) Obvious effect rate: The systolic pressure (systolic blood pressure, SBP) control at 120-140 mmHg level, diastolic pressure (diastolic blood pressure, DBP) control at $70-85 \mathrm{mmHg}$ levels; ii) effective rate: SBP decline is $>10 \mathrm{mmHg}$ and DBP control at $60-85 \mathrm{mmHg}$ level; and iii) null and void: None of these standards were reached. Total effective rate of blood pressure control $=$ obvious effect rate + effective rate. 
Therapeutic method. The Control group was orally administered $75 \mathrm{mg} /$ day amlodipine besylate (H20066824; Beijing Wansheng Pharmaceutical Co., Ltd.). Treatment group orally administered $5 \mathrm{mg} /$ day amlodipine besylate combined with $75 \mathrm{mg} /$ day acupoint application of traditional Chinese medicine nursing (acupoint Neiguan, Yongquan and Sanyinjiao). The prescription of AA-TCM nursing is composed of $2 \mathrm{~g}$ psoralen, $0.6 \mathrm{~g}$ Wu Zhuyu, $0.6 \mathrm{~g}$ cinnamon, $2 \mathrm{~g}$ cooked aconite, $0.6 \mathrm{~g}$ clove, $1 \mathrm{~g}$ ephedra, $2 \mathrm{~g}$ mulberry and $2 \mathrm{~g}$ Herba Epimedium. When applied to patients, it is advisable to adjust the amount of ginger juice into thick paste and make it into a cone shaped medicinal cake with a diameter of $2 \mathrm{~cm}$. Operation method: The patients' acupoints (Neiguan, Yongquan and Sanyinjiao acupoints) were fully exposed by the nurse and routinely disinfected with $75 \%$ alcohol. After that, the medicine cakes were placed in the center of the non-woven cloth application $(6 \times 7 \mathrm{~cm})$ and fixed on the acupoints for 4-6 h, once a day for 2 weeks.

Animals and treatment. A total of 40 Male SHR rats $(200 \pm 20 \mathrm{~g}$, 6 weeks of age) were purchased from Ji'nan PengYue Experimental Animal Breeding Co., Ltd. [Zhangqiu, Jinan, China; quality certificate number, SCXK (Lu) 20140007]. All animals were housed in a controlled environment at $22 \pm 1^{\circ} \mathrm{C}$ and relative humidity $(60 \pm 10) \%$ under a 12 -h dark/light cycle with free access to food and water. Animal experiments were performed by following the National institute of Health (NIH) guidelines (20). All experimental procedures were approved by the Animal Care and Use Committee of Yantai Hospital of Tradition Chinese Medicine and performed in strict accordance with the People's Republic of China Legislation Regarding the Use and Care of Laboratory Animals.

After two weeks of acclimatisation, the animals were randomly divided into 4 groups, including the Sham operation control group which underwent opening of the abdominal cavity to expose the kidney, but no nephrectomy (Sham group), the Model group, Amlodipine besylate treatment ( $1 \mathrm{mg} / \mathrm{kg} / \mathrm{day})$ group (NC group) and amlodipine besylate $(1 \mathrm{mg} / \mathrm{kg} /$ day) combined with acupoint sticking therapy group (Treatment group). The acupoints of the rat acupoint application method were selected to be the same as those of the patient. The prepared application was placed on the above acupoints. If the rats bit the application this was fixed with medical tape. One acupoint was treated with one Chinese medicine patch. Each treatment lasted for $1 \mathrm{~h}$ and was performed every other day for 6 weeks. The experimental diets were given ad libitum for 8 weeks in the form of pellets and the animals were sacrificed following an overnight fast. Then, blood $(0.5 \mathrm{ml})$ was harvested and stored at $4^{\circ} \mathrm{C}$. The kidney tissue was collected and stored at $-80^{\circ} \mathrm{C}$ for further analysis. All rats were anesthetized with $1 \%$ pentobarbital sodium $(30 \mathrm{mg} / \mathrm{kg})$. Following strict disinfection, $2 / 3$ of the right kidney was resected. The treated rats were then given access to normal diet and water. At age 9 weeks, the above rats were anesthetized, disinfected again and the whole left kidney was removed to establish an SHR-CRF animal model with only $1 / 3$ of a kidney. After operation, the normal diet and drinking water were restored for 1 week. The rats were fed alone in individual cages. At the age of 10 weeks, rats were fed intragastrically to observe the general condition of rats. The rats were then put into a clean metabolic cage.
Following access to normal drinking water, $24 \mathrm{~h}$ urine was collected and the volume was measured. T blood pressure of rats was measured by a non-invasive blood pressure meter. SPB and DBP were measured every 2 weeks. Rats were raised to 20 weeks age, after which they received intraperitoneal injection of $3 \%$ pentobarbital sodium $(40 \mathrm{mg} / \mathrm{kg})$. The rats were anesthetized and blood biochemical test was performed after the carotid artery was excised. Part of the renal tissue was taken to fix for $24 \mathrm{~h}$ at room temperature in $10 \%$ formalin. The tissues were paraffin was embedded prior to hematoxylin and eosin (H\&E) staining and immunohistochemistry, and then transferred to $-80^{\circ} \mathrm{C}$ refrigerator following freezing with liquid nitrogen, and used for reverse transcription quantitative polymerase chain reaction (RT-qPCR) and western blotting detection.

Biochemical analysis. Blood samples were placed at room temperature until serum appeared in the upper layer. Serum was separated via centrifugation at $1,000 \mathrm{x} \mathrm{g}$ and $4^{\circ} \mathrm{C}$ for $10 \mathrm{~min}$, and the supernatant was collected and maintained at $-20^{\circ} \mathrm{C}$ for preservation. The levels of serum urea nitrogen (BUN), creatinine (CRE), total protein (TP) and albumin (ALB) were determined by an automatic biochemical analyzer. Urine protein (UP) was determined using a UP ELISA Kit (cat. no. DRE9802Ra; Shanghai Lianshuo Biological Technology Co., Ltd., Shanghai, China). The contents of urea nitrogen, urine creatinine, $\mathrm{N}$-acetyl-Glucosaminidase (NAG) in urine, and creatinine clearance $(\mathrm{Ccr})$ were measured by an automatic biochemical analyzer. Endogenous creatinine clearance $(\mathrm{ml} / \mathrm{kgB} . \mathrm{W} . / \mathrm{min})=$ concentration of urinary creatinine $\mathrm{x} 24 \mathrm{~h}$ urine volume/serum creatinine concentration $(\mathrm{ml}) / 24 / 60 / 1,000 \mathrm{x}$ body weight $(\mathrm{g})$.

$H \& E$ staining. After being sacrificed, the renal tissues of the rats were embedded in paraffin and fixed in $10 \%$ formalin for at least $24 \mathrm{~h}$ at room temperature. The samples were then cut into $5-\mu \mathrm{m}$ pieces and fixed on slides according to the routine procedure. Renal sections were stained with hematoxylin (Beijing Solarbio Science \& Technology Co., Ltd.) for 5 min at room temperature and washed with tap water. They were differentiated by hydrochloric acid ethanol for $30 \mathrm{sec}$ and soaked for 15 min with water, then placed in eosin dye solution (Beijing Solarbio Science \& Technology Co., Ltd.), dehydrated for $2 \mathrm{~min}$. Histopathological changes were observed under an Olympus microscope (BX51 Olympus, magnification, 200; Olympus Corporation, Tokyo, Japan).

Immunohistochemistry assay. Immunohistochemistry staining of tissue slices for VEGF and matrix metallopeptidase 9 (MMP9) were performed using a VEGF primary antibody (1:1,600; cat. no. 9698; Cell Signaling Technology, Inc., Danvers, MA, USA) and an MMP9 primary antibody (1:325; cat. no. 13667; Cell Signaling Technology, Inc.) in a humidified chamber at $4^{\circ} \mathrm{C}$ overnight. After rewarming, the tissue slices were incubated with goat horseradish peroxidase-conjugated secondary antibodies against rabbit immunoglobulin (Ig)G (1:1,000; cat. no. 7074; Cell Signalling Technology, Inc.), then dyed with DAB and dehydrated. It was observed under x400 optical microscope (Olympus Corporation). The positive staining was evaluated by a hierarchical system. The score 
was defined as: 0 , no staining; 1 , mild staining; 2 , moderate staining; 3 , strong staining. In order to maintain the consistency of the scores, each part was scored by two inexperienced tissue scientists, and the average value was calculated.

Detection of expression level of associated mRNA by RT-qPCR. The total RNA was extracted using the TRIzol kit [Invitrogen; Thermo Fisher Scientific, Inc., Waltham, MA, USA; the purity of RNA was qualified by optical density (OD)260/OD280 between 1.8-2.0]. RT was performed with the SuperScript ${ }^{\mathrm{TM}}$ III First-Strand short SuperMix for qPCR retrovirus kit (cat. no. 11752250; Thermo Fisher Scientific, Inc.) according to the manufacturer's protocol. The temperature protocol was as follows: $50^{\circ} \mathrm{C}$ for $30 \mathrm{~min}, 85^{\circ} \mathrm{C}$ for $5 \mathrm{~min}$ and termination at $4^{\circ} \mathrm{C}$. Master EP realplex2 (Eppendorf, Hamburg, Germany) was used to conduct quantitative PCR (conditions: $94^{\circ} \mathrm{C}$ for $3 \mathrm{~min}, 94^{\circ} \mathrm{C}$ for $30 \mathrm{sec}, 55^{\circ} \mathrm{C}$ for $30 \mathrm{sec}, 30$ cycles). The relative expression of mRNA were calculated by $2^{-\Delta \Delta \mathrm{Cq}}$ method (21), and the $\beta$-actin mRNA was used as a reference to calculate the relative expression levels. Primers (Sangon Biotech Co., Ltd., Shanghai, China) were listed in Table I.

Western blotting. Total proteins were extracted from tissues according to the whole protein extraction kit (cat. no. BC3710; Beijing Solarbio Science \& Technology Co., Ltd.). The renal tissues were were mechanically crushed and centrifuged at $3,000 \mathrm{x} \mathrm{g}$ and $4^{\circ} \mathrm{C}$ for $10 \mathrm{~min}$. The supernatant was taken and the protein concentrations of the renal tissues were measured by the bicinchoninic acid kit (Beijing Solarbio Science \& Technology Co., Ltd.). A total of $30 \mu \mathrm{g}$ samples protein and $10 \%$ SDS-PAGE were mixed 1:1. The protein denaturation by heating $5 \mathrm{~min}$ at $95^{\circ} \mathrm{C}$ The protein samples were transferred to polyvinylidene difluoride membrane (Merck KGaA, Darmstadt, Germany) under the semi drying condition of $20 \mathrm{~V}$ for $30 \mathrm{~min}$ (Bio-Rad Laboratories, Inc., Hercules, CA, USA). The membranes were washed and blocked with $5 \%$ evaporated milk for $2 \mathrm{~h}$ at room temperature. Primary antibodies were added, including rabbit anti PI3K (1:1,000; cat. no. 4249), p-PI3K (1:1,000; cat. no. 4228), p-AKT $(1: 2,000$; cat. no. 4060), AKT (1:1,000; cat. no. 4691), p-NF-кB p65 (1:1,000; cat. no. 8214), NF-кB p65 (1:1,000; cat. no. 8242) and rabbit anti $\beta$-actin antibody (1:1,000; cat. no. 4970; all from Cell Signaling Technology, Inc.) and incubated at $4^{\circ} \mathrm{C}$ overnight. After washing 3 times, goat anti-rabbit $\mathrm{IgG}$-horseradish peroxidase (1:1,000; ABIN101988; antibodies-online $\mathrm{GmbH}$, Aachen, Germany) was added and incubated for $1 \mathrm{~h}$. The results were observed and recorded by Roche Elecsys-2010 chemiluminescence (Roche Diagnostics, Basel, Switzerland). Protein expression level were normalized according to the $\beta$-actin and quantified by Image J 1.48u (National Institutes of Health, Bethesda, MD, USA) software.

Statistical analysis. Data were analysed by SPSS 19.0 statistical software (IBM Corps, Armonk, NY, USA). Each experiment was repeated three times. The results of DBP, SBP, each situation and symptom score following the treatment of hypertensive patients and rats were expressed as the mean \pm standard deviation. A t-test was used for data analysis between the two groups. One-way analysis of variance was used for data analysis among multiple groups. The least signif-
Table I. The primer sequences used in the present study.

Gene Sequence

PI3K Forward: 5'-CAATACTTGATGTGGCTGAC-3' Reverse: 5'-CAATACTTGATGTGGCTGAC-3'

AKT Forward: 5'-TTTATTGGCTACAAGGAACG-3' Reverse: 5'-AGTCTGAATGGCGGTGGT-3'

ET-1 Forward: 5'-TCTCTGCTGTTTGTGGCTTTC-3' Reverse: 5'-TCGGAGTTCTTTGTCTGCTTG-3'

$\beta$-actin Forward: 5'-TCAGGTCATCACTATCGGCAAT-3' Reverse: 5' - AAAGAAAGGGAGTAAAACGCA-3'

AKT, protein kinase B; ET, endothelin; PI3K, phosphoinositide 3-kinase.

icant difference test was used for subsequent analysis. $\mathrm{P}<0.05$ was considered to indicate a statistically significant difference.

\section{Results}

Changes of blood pressure in hypertensive patients with renal failure. There was no significant difference in DBP and SBP between the two groups prior to treatment $(\mathrm{P}>0.05)$. Following treatment, the difference between the two groups was statistically significant $(\mathrm{P}<0.05)$. The results proved that the treatment group was better than the control group in reducing DBP and SBP (Table II). The results demonstrated that amlodipine besylate combined with AA-TCM nursing has obvious therapeutic effect on renal failure and hypertension.

Clinical efficacy of treatment of renal failure patients with hypertension. As presented in Table III, there were 30 patients with renal failure in the Control group. The average age of the 25-45 year old patients was $38.60 \pm 6.58$, including 4 males $(80.0 \%)$ and 1 female $(20.0 \%)$. The average age of the 46-65 year old patients was $58.43 \pm 6.09$, including 8 males (57.1\%) and 6 females (42.9\%). The average age of the 66-85 year old patients was $73.91 \pm 5.80$, including 3 males $(27.3 \%)$ and 8 females $(72.7 \%)$. Additionally there were 30 patients with renal failure in the treatment group. The average age of 25-45 year old patients was $35.75 \pm 9.57$, including 3 males $(75.0 \%)$ and 1 female $(25.0 \%)$. The average age of 46-65 year old patients was 59.8 \pm 3.97 , including 4 males (26.7\%) and 11 females (73.3\%). The average age of 66-85 year old patients was $74.09 \pm 4.06$, including 4 males $(36.4 \%)$ and 7 females $(63.6 \%)$. The results demonstrated that the treatment effect was independent of age and sex $(\mathrm{P}>0.05)$.

Total effective rate of hypertension in renal failure. After treatment, the two groups' blood pressures were significantly reduced. The total effective rate in the Control group was $56.7 \%$, and the total effective rate in the Treatment group was $80 \%$. The difference between the two groups was statistically significant $(\mathrm{P}<0.05$; Table IV).

The general condition of the rats. The rats in the Sham group had glossy hair, quick reactions, a normal intake of water and 
Table II. Changes in blood pressure before and after treatment in two groups.

\begin{tabular}{lccccc}
\hline & \multicolumn{2}{c}{ Control group } & & \multicolumn{2}{c}{ Treatment group } \\
\cline { 2 - 3 } Index & Before treatment & After treatment & & Before treatment & After treatment \\
\hline SBP & $159.10 \pm 11.29$ & $139.83 \pm 11.93^{\mathrm{a}}$ & $162.07 \pm 14.74$ & $133.43 \pm 10.52^{\mathrm{a}, \mathrm{b}}$ \\
DBP & $93.60 \pm 11.97$ & $84.37 \pm 10.01^{\mathrm{a}}$ & & $90.67 \pm 9.81$ & $79.53 \pm 8.52^{\mathrm{a}, \mathrm{b}}$ \\
\hline
\end{tabular}

${ }^{\mathrm{a}} \mathrm{P}<0.05$ vs. before treatment, ${ }^{\mathrm{b}} \mathrm{P}<0.05$ vs. after treatment in the Control group. SBP, systolic bloodpressure; DBP, diastolic blood pressure.

Table III. Effect of the treatment group on hypertensive patients with renal failure.

\begin{tabular}{|c|c|c|c|c|c|}
\hline & Obvious effective (\%) & Effective (\%) & No effective (\%) & Total effective rate $(\%)$ & P-value \\
\hline Control group & & & & & 0.09 \\
\hline \multicolumn{6}{|l|}{ Age } \\
\hline $25-45$ & $1(20.0)$ & $0(0.0)$ & $4(80.0)$ & $1(20.0)$ & \\
\hline $46-65$ & $6(42.9)$ & $2(14.3)$ & $6(42.9)$ & $8(57.1)$ & \\
\hline $66-85$ & $3(30.0)$ & $4(40.0)$ & $4(40.0)$ & $7(70.0)$ & \\
\hline Sex & & & & & 1.000 \\
\hline Male & $4(26.7)$ & $4(26.7)$ & $7(46.7)$ & $8(72.7)$ & \\
\hline Female & $6(40.0)$ & $2(13.3)$ & $7(46.7)$ & $8(53.3)$ & \\
\hline Treatment group & & & & & 0.216 \\
\hline \multicolumn{6}{|l|}{ Age } \\
\hline $25-45$ & $2(50.0)$ & $0(0.0)$ & $2(50.0)$ & $2(50.0)$ & \\
\hline $46-65$ & $11(73.3)$ & $1(6.7)$ & $3(20.0)$ & $12(80.0)$ & \\
\hline $66-85$ & $7(63.6)$ & $3(27.3)$ & $1(9.1)$ & $10(90.9)$ & \\
\hline Sex & & & & & 0.449 \\
\hline Male & $7(63.6)$ & $1(9.1)$ & $3(27.3)$ & $8(72.7)$ & \\
\hline Female & $13(68.4)$ & $3(15.8)$ & $3(15.8)$ & $16(84.2)$ & \\
\hline
\end{tabular}

Table IV. The efficacy of treatment in two groups.

\begin{tabular}{|c|c|c|c|c|c|}
\hline Group & Obvious effective (\%) & Effective (\%) & No effective $(\%)$ & Total effective rate $(\%)$ & P-value \\
\hline Control group & $9(30.0)$ & $7(23.3)$ & $14(46.7)$ & $16(53.3)$ & 0.028 \\
\hline Treat group & $20(66.7)$ & $4(13.3)$ & $6(0.2)$ & $24(80.0)$ & \\
\hline
\end{tabular}

body weight. In the Model group, the rats were depressed, dull fur and sluggish, slow in activity, slow in appetite and slow in growth. Compared with the Model group, the time of occurrence of symptoms in the NC group and Treatment group was delayed to varying degrees, and symptoms improved significantly. Compared with the NC group, the symptoms of the Treatment group improved slightly.

Effect of amlodipine besylate combined with AA-TCM nursing on blood pressure in hypertensive rats with renal failure. As presented in Fig. 1, the systolic and diastolic pressure of rats in Model group was significantly increased compared with the Sham group $(\mathrm{P}<0.05)$. Compared with the Model group, the systolic and diastolic pressure of the NC group and Treatment group decreased significantly at the beginning of the treat- ment (12 weeks age), and there was a significant difference $(\mathrm{P}<0.05)$. Compared with the NC group, the blood pressure of the Treatment group decreased slightly. The results demonstrated that amlodipine besylate combined AA-TCM nursing exhibited significant antihypertensive effects on renal failure hypertension.

Effect of amlodipine besylate combined with AA-TCM nursing on blood biochemical indexes in hypertension rats with renal failure. As presented in Fig. 2, compared with the Sham group, the concentration of TP and ALB in the Model group was not significantly different, but the concentration of BUN and CRE increased significantly $(\mathrm{P}<0.05)$. Compared with the Model group, there was no significant difference in the concentrations of TP and ALB between the NC group and the 

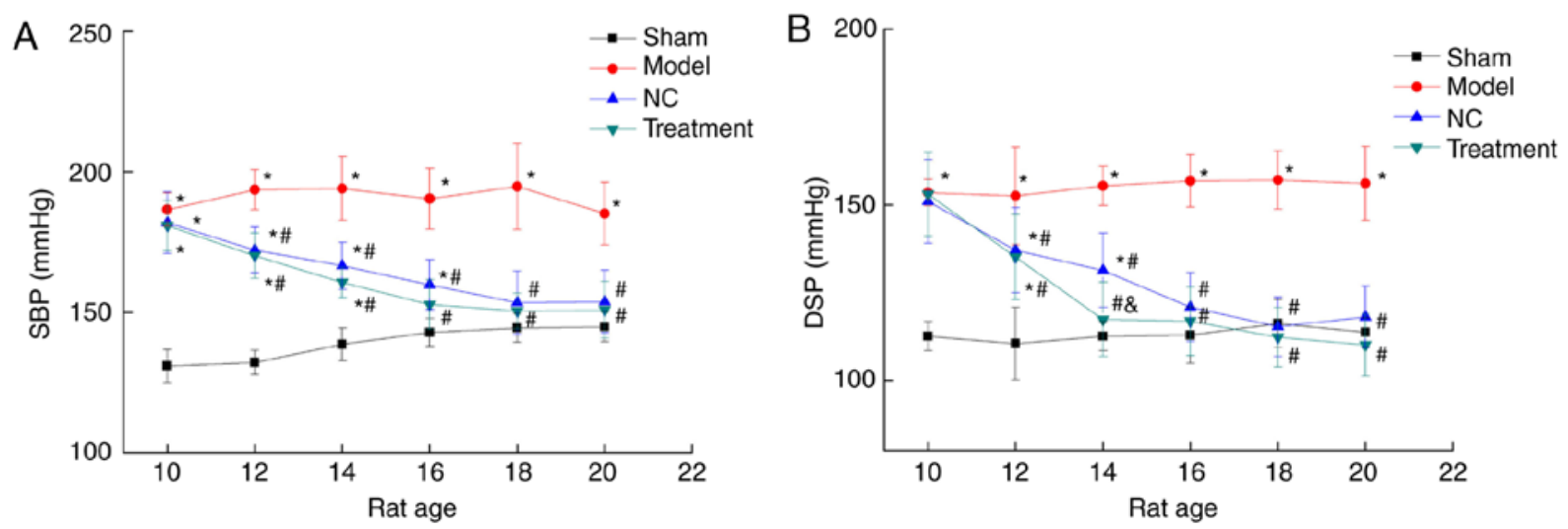

Figure 1. Effect of amlodipine besylate combined with acupoint application on blood pressure in hypertension rats with renal failure. (A) Changes in systolic blood pressure in rats. (B) changes in DBP in rats. ${ }^{*} \mathrm{P}<0.05$ vs. the Sham group, ${ }^{\#} \mathrm{P}<0.05$ vs. the Model group. ${ }^{\circledR} \mathrm{P}<0.05$ vs. the NC group. NC, negative control; DBP, diastolic blood pressure; SBP, systolic blood pressure.
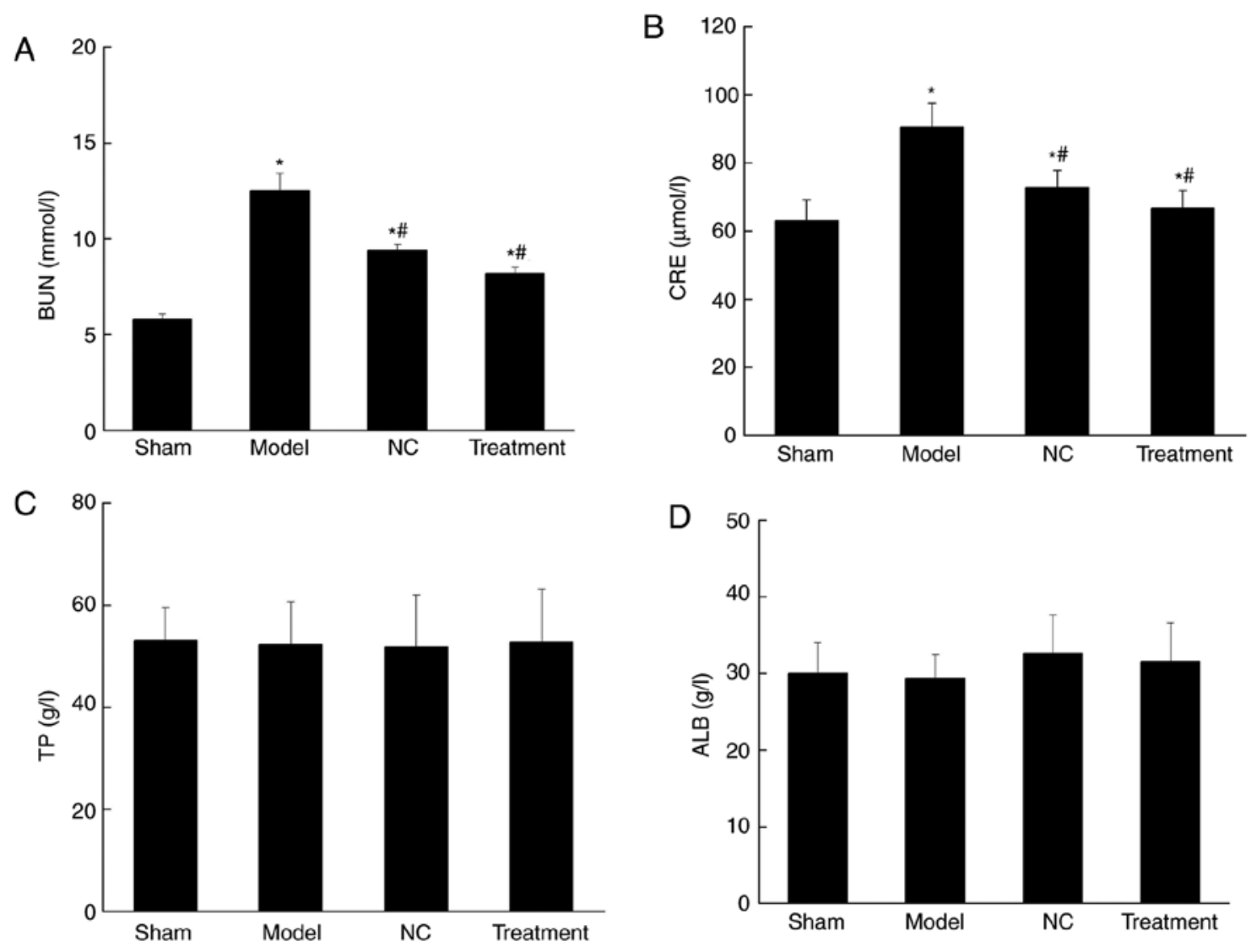

Figure 2. Effect of amlodipine besylate combined with acupoint application on blood biochemical indexes in hypertension rats with renal failure. (A) The concentrations of BUN. (B) The concentration of CRE. (C) The concentration of TP. (D) The concentration of ALB. "P<0.05 vs. the Sham group and ${ }^{~} \mathrm{P}<0.05$ vs. the Model group. NC, negative control; CRE, carbapenem-resistant Enterobacteriaceae; TP, total protein; BUN, blood urea nitrogen; ALB, albumin.

Treatment group following treatment, but the concentrations of BUN and CRE decreased significantly $(\mathrm{P}<0.05)$. Compared with the NC group, the concentrations of BUN and CRE in the Treatment group decreased slightly. The results demonstrated that amlodipine besylate combined with AA-TCM nursing had no effect on TP and ALB, and could increase and decrease the concentration of BUN and CRE in the serum of rats.

Effect of amlodipine besylate combined with AA-TCM nursing on urine index in hypertension rats with renal failure. As presented in Fig. 3, compared with the Sham group, the content of UP, BUP, CRE and NAG in the Model group increased significantly, and Ccr decreased significantly $(\mathrm{P}<0.05)$. Compared with the Model group, the contents of UP, BUP, CRE and NAG in NC group and the Treatment group were significantly decreased, while $\mathrm{Ccr}$ was significantly increased $(\mathrm{P}<0.05)$. Compared with the NC group, the urine index of group Treatment was closer to the normal value. The results demonstrated that amlodipine besylate combined with AA-TCM nursing could enhance the clearance rate of serum creatinine in rats. 

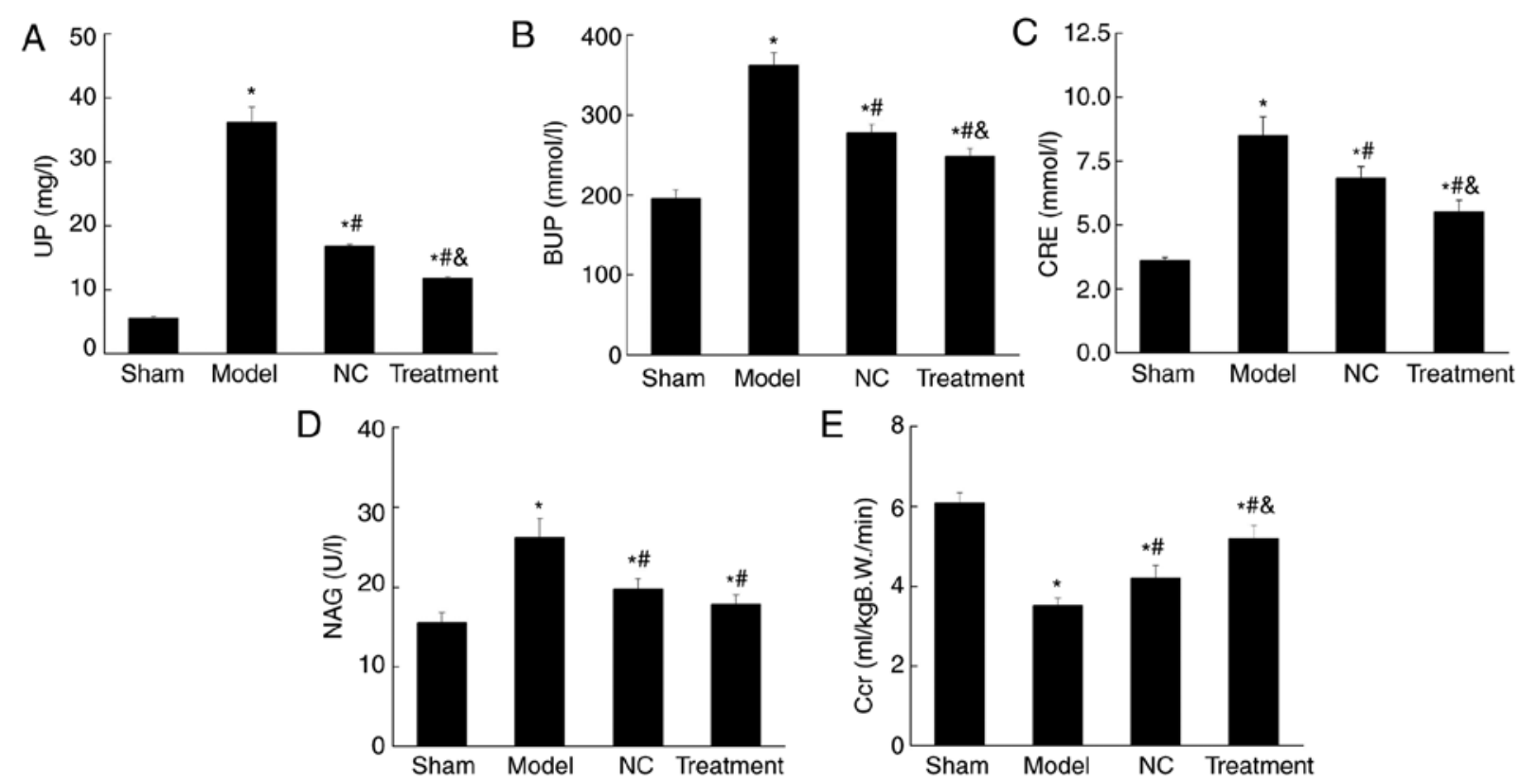

Figure 3. Effect of amlodipine besylate combined with acupoint application on urine index in hypertension rats with renal failure. (A) The content of UP. (B) The content of BUP. (C) The content of CRE. (D) The content of NAG. (E) Ccr. ${ }^{*} \mathrm{P}<0.05$ vs. the Sham group, ${ }^{*} \mathrm{P}<0.05$ vs. the Model group and ${ }^{\&} \mathrm{P}<0.05$ vs. the NC group. NC, negative control; UP, urine protein; BUP, blood urea protein; NAG, N-acetyl- $\beta$-D-glucosaminidase; CRE, carbapenem-resistant Enterobacteriaceae; Crr Creatinine clearance rate.

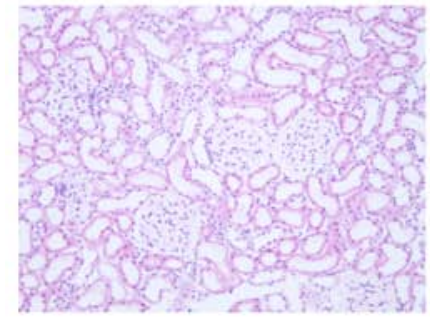

Sham

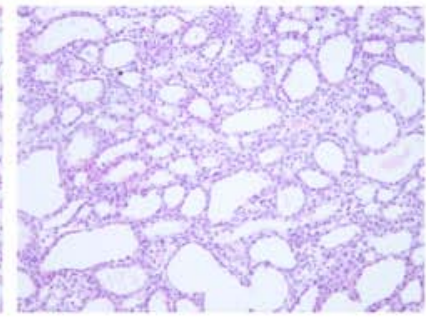

Model

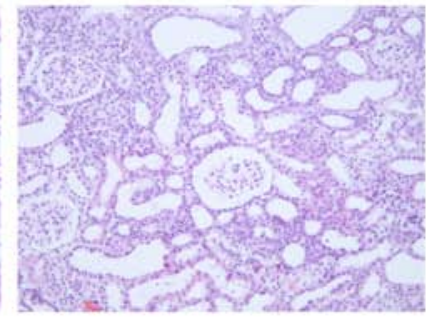

NC

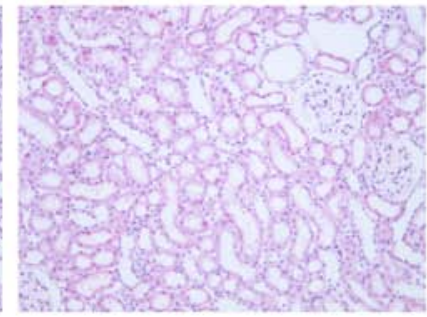

Treatment

Figure 4. Pathological alterations of renal tissue in rats with hematoxylin and eosin staining (magnification, x200). NC, negative control.

Pathological changes of renal tissue in rats. As presented in Fig. 4, the glomerular, renal tubules and interstitial tissue in the Sham group exhibited a normal arrangement and distribution. In the Model group, the following observations were made: The wall of glomeruli was thickened, capillary dilation or occlusion, partial glomerular segmental or spherical sclerosis, mesangial cell proliferation, renal tubules as flat epithelial cells, vacuolating part of epithelial cells, part of renal tubules dilated, interstitial cells and fibroblasts, and interstitial collagen in interstitial cells. While, glomeruli, tubulointerstitial and interstitial lesions in the $\mathrm{NC}$ group and Treatment group were relieved. Compared with the NC group, the pathological changes of Treatment group were improved.

Effect of amlodipine besylate combined with AA-TCM nursing on VEGF and MMP9 in renal tissue of hypertensive rats with renal failure. As presented in Fig. 5, VEGF was expressed in the renal interstitium and glomerulus, and MMP9 was expressed in the cytoplasm of renal tubular epithelial cells. Compared with the Model group, the scores of VEGF and MMP9 in the NC group and Treatment group decreased significantly $(\mathrm{P}<0.05)$. Compared with the $\mathrm{NC}$ group, the contents of VEGF and MMP9 in the Treatment group were significantly decreased $(\mathrm{P}<0.05)$.

Effects of amlodipine besylate combined with AA-TCM nursing on the PI3K/AKT pathway associated $m R N A$ in hypertensive rats with renal failure. As presented in Fig. 6, compared with the Sham group, the mRNA expression of PI3K and AKT in the Model group was significantly decreased, and the mRNA expression of ET-1 increased significantly $(\mathrm{P}<0.05)$. Compared with the Model group, the mRNA expression of PI3K and AKT in the NC group and Treatment group increased significantly in hypertensive rats with renal failure, and the mRNA expression of ET-1 was significantly decreased $(\mathrm{P}<0.05)$. Compared with the NC group, the mRNA expression of PI3K in the Treatment group was significantly increased $(\mathrm{P}<0.05)$, but the mRNA expression of AKT and ET-1 were not significantly changed $(\mathrm{P}>0.05)$.

Effects of amlodipine besylate combined with AA-TCM nursing on PI3K/AKT pathway associated proteins in hypertensive rats with renal failure. As presented in Fig. 7, compared with the Sham group, the expression of p-PI3K 

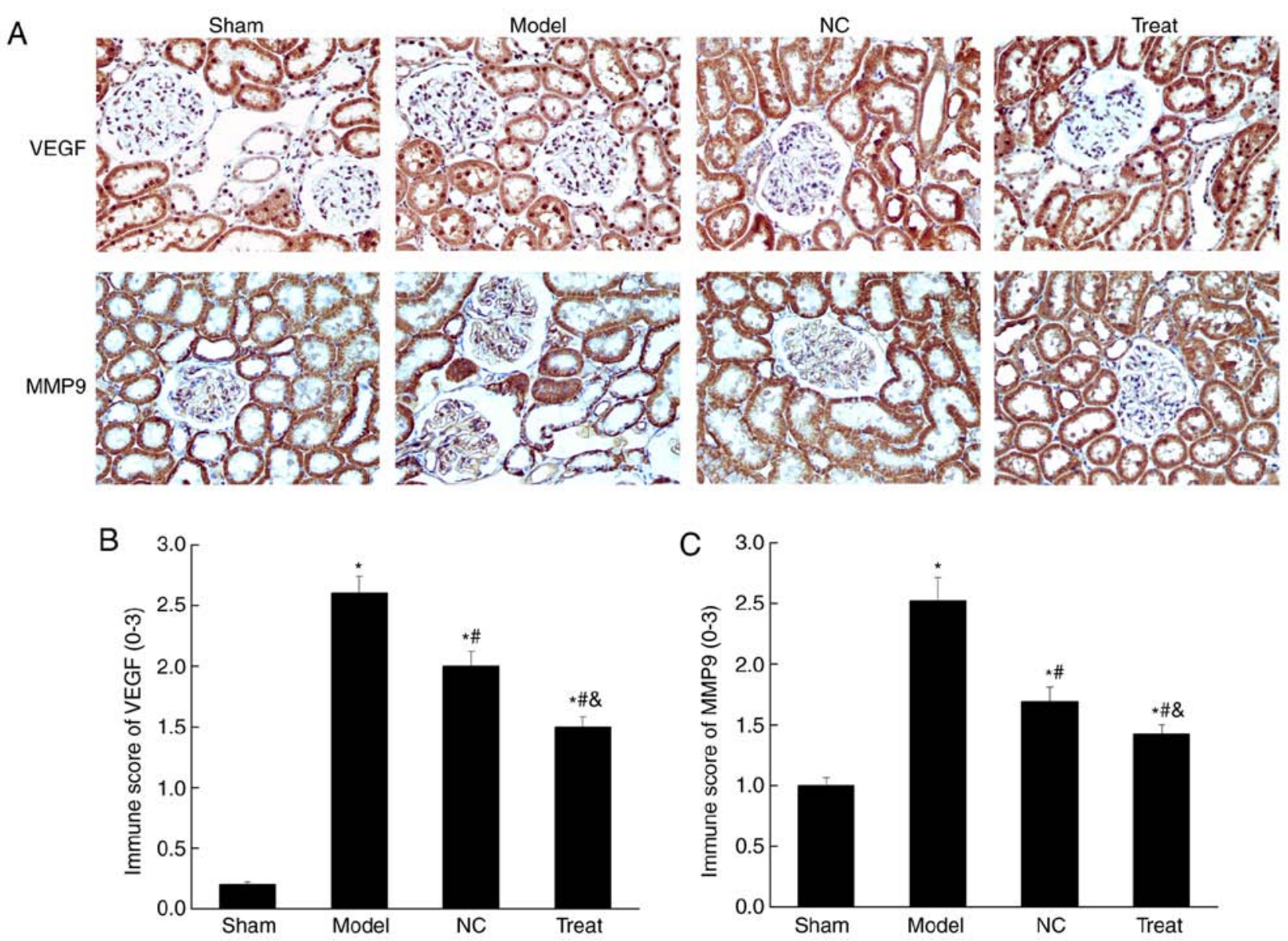

Figure 5. Effect of amlodipine besylate combined acupoint application on VEGF and MMP9 in renal tissue of hypertensive rats with renal failure. (A) Immunohistochemistry. (B) VEGF immunohistochemical score. (C) MMP9 immunohistochemical score. ${ }^{*} \mathrm{P}<0.05$ vs. the Sham group, ${ }^{\prime \prime} \mathrm{P}<0.05$ vs. the Model group and ${ }^{\&} \mathrm{P}<0.05$ vs. the NC group. VEGF, vascular endothelial growth factor; MMP, matrix metalloproteinase; NC, negative control.
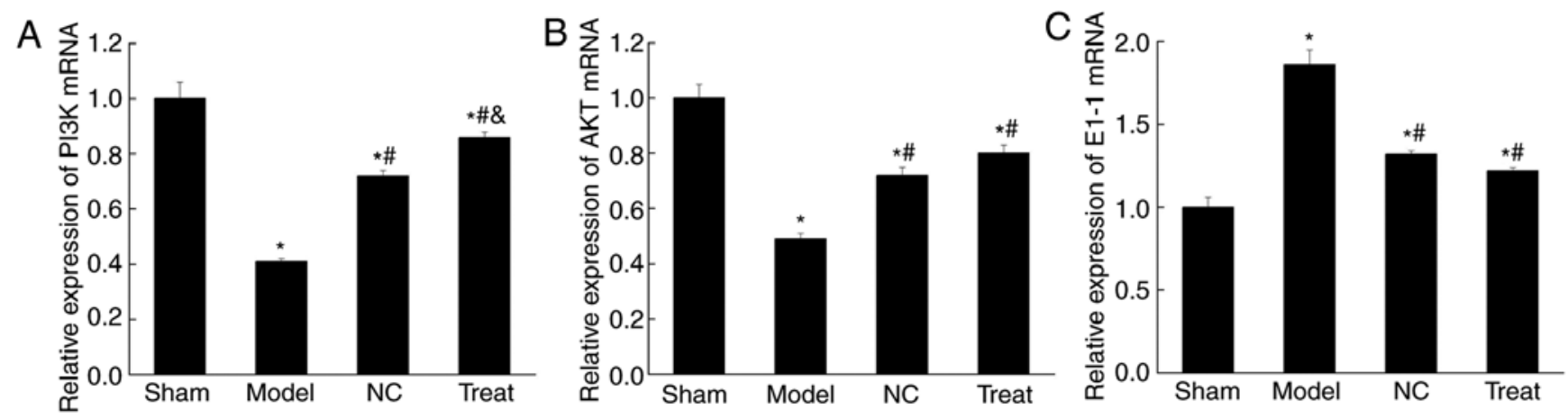

Figure 6. Effect of amlodipine besylate combined acupoint application on PI3K/AKT pathway related mRNA in hypertensive rats with renal failure. (A) The mRNA expression of PI3K. (B) The mRNA expression of AKT. (C) The mRNA expression of ET-1 mRNA. "P $<0.05$ vs. the Sham group, ${ }^{*} \mathrm{P}<0.05$ vs. the Model group, ${ }^{\&} \mathrm{P}<0.05$ vs. the $\mathrm{NC}$ group. AKT, protein kinase B; PI3K, phosphoinositide 3-kinase; NC, negative control.

and $\mathrm{p}-\mathrm{AKT} / \mathrm{AKT}$ proteins in hypertensive rats with renal failure in the Model group was significantly decreased, and the expression of $\mathrm{p}-\mathrm{NF}-\mathrm{\kappa B}$ p65/NF- $\mathrm{KB}$ p65 protein increased significantly $(\mathrm{P}<0.05)$, and the expression of PI3K and p-AKT/AKT proteins in the NC group and Treatment group of hypertensive rats with renal failure was significantly increased and $\mathrm{p}-\mathrm{NF}-\kappa \mathrm{B}$ p65/NF- $\mathrm{BB}$ p 65 protein decreased significantly compared with those in the Model group $(\mathrm{P}<0.05)$. Compared with the NC group, the expression of p-PI3K/PI3K protein in the Treatment group were significantly increased, and the expression of $\mathrm{p}-\mathrm{AKT} / \mathrm{AKT}$ and $\mathrm{p}-\mathrm{NF}-\kappa \mathrm{B}$ p65/NF- $\kappa \mathrm{B}$ p65 protein were significantly decreased $(\mathrm{P}<0.05)$.

\section{Discussion}

Renal hypertension is a secondary hypertensive condition, mainly due to renal hypertension and renal artery disease (22). This disease is mainly characterized by degeneration of glomeruli, interstitial tissue and connective tissue hyperplasia, renal tubule atrophy, small renal artery stenosis or non-specific 

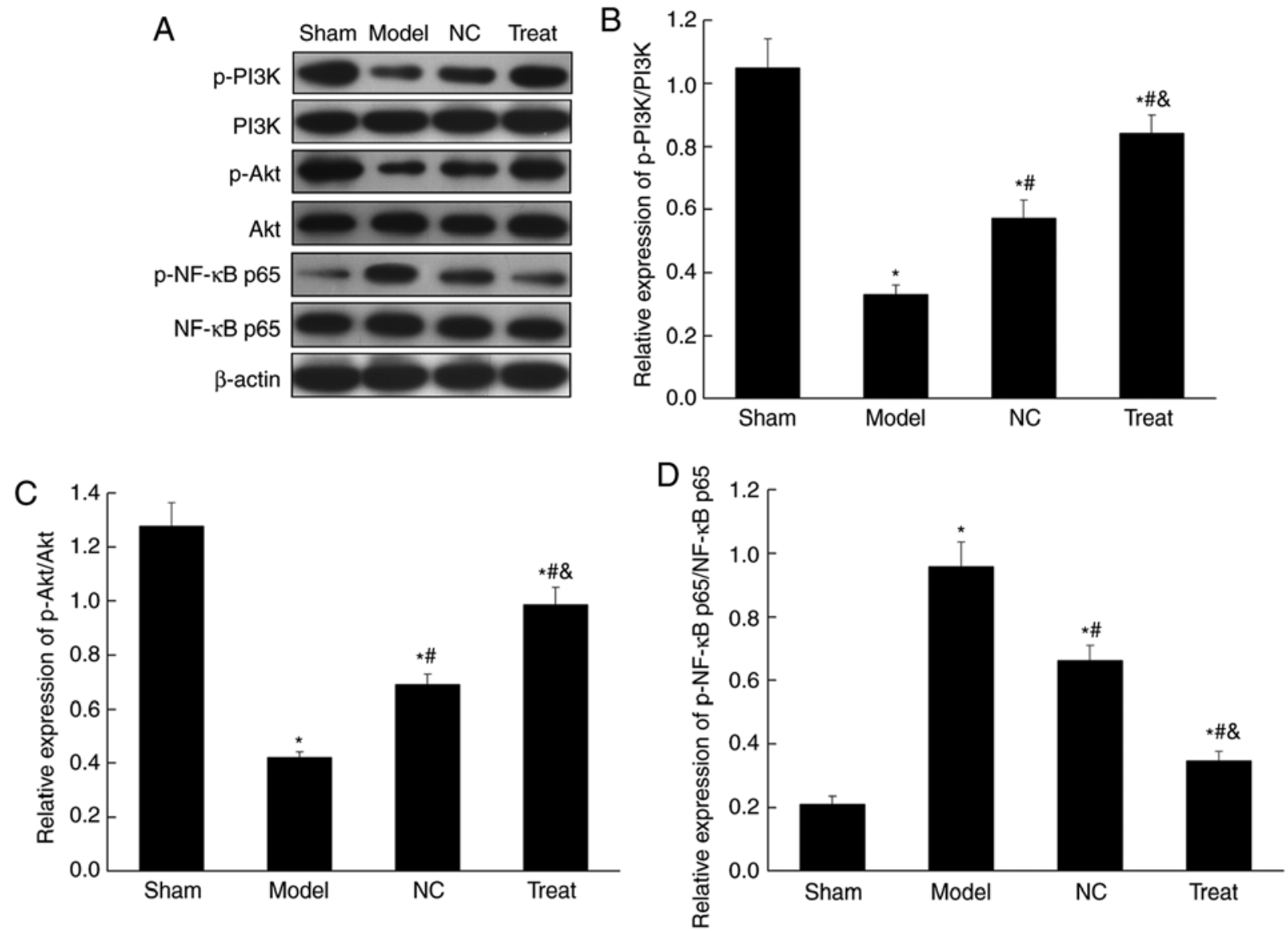

Figure 7. Effect of amlodipine besylate combined acupoint application on PI3K/AKT pathway related proteins in hypertensive rats with renal failure. (A) Western blotting. (B) The expression of p-PI3K/PI3K protein. (C) The expression of p-AKT/AKT protein. (D) The expression of p-NF- $\mathrm{kB}$ p65/NF- $\mathrm{kB}$ p 65 protein. ${ }^{*} \mathrm{P}<0.05$ vs. the Sham group, ${ }^{\prime \prime} \mathrm{P}<0.05$ vs. the Model group and ${ }^{\&} \mathrm{P}<0.05$ vs. the NC group. $\mathrm{P}$, phosphorylated; AKT, protein kinase B; p-PI3K, phosphorylated phosphoinositide 3-kinase; NF, nuclear factor.

Takayasu arteritis resulting in substantial damage to the kidney and lack of blood supply (23). Mucous myofibrillar hyperplasia in the middle layer of renal artery wall forms most small aneurysms, thus the inner wall of renal arterioles protrude like beads, resulting in segmental stenosis of renal artery (24-26). Hypertension can cause kidney damage, and the two will promote each other, which will further develop the disease. Therefore, renal hypertension should actively be treated (27).

The negative inotropic effects of amlodipine besylate were observed in vitro, but not in animal experiments (28). This product does not affect the concentration of plasma calcium. Clinical trials have confirmed its antihypertensive effect. Prolonged use did not cause changes in heart rate or plasma catecholamine (29). The effect of depressurization is gradual and the difference between peak and valley value is not large. The effect of hypotension is associated with the dose (30). The change in amplitude is associated with blood pressure prior to treatment. The moderate hypertension patients exhibited improvement compared with mild hypertension patients following administration, whereas the patients with normal blood pressure have no obvious effects (31). Amlodipine benzoate can directly affect diastolic vascular smooth muscle and have an antihypertensive effect. This product relieves angina by dilating peripheral arteries, reducing peripheral resistance, reducing myocardial oxygen consumption and expanding the coronary arteries of normal and ischemic areas to improve the myocardial supply of patients with coronary spasm (32). Acupoint sticking therapy is a noninvasive pain acupoint therapy based on meridian theory of traditional Chinese medicine (TCM), which is used to treat diseases. It is an important component of TCM therapeutics, which are a set of unique and effective treatment methods performed by the working people of China in the struggle with disease. The mechanism of acupoint sticking therapy is complicated. According to the theory of viscera-meridian in TCM, acupoints are closely associated with viscera through meridians, which not only reflect the physiological or pathological functions of each viscera, but also is an effective stimulating point for the treatment of diseases of the viscera (33).

The PI3K/serine threonine kinase (V-AKT murine thymoma viral oncogene homolog, AKT) pathway is the key signal transduction pathways in eukaryotic cells (34). It serves an important role in biological activities including apoptosis, metabolism, proliferation and differentiation. The active form of AKT in the body is phosphorylated at 308th site threonine and 473rd site serine. Thr308 phosphorylation serves a role in stabilizing the activation state of AKT, so the detection of the expression of ser473 phosphorylation AKT protein is a marker of the degree of activation of the PI3K/AKT pathway $(35,36)$. The PI3K/AKT signaling pathway is closely associated with renal disease. It is demonstrated that regulating the activity 
of PI3K/AKT can affect the proliferation of mesangial cells and the secretion of the extracellular matrix. In the unilateral ureteral obstruction rat model, the PI3K/AKT signaling pathway was involved in the process of renal interstitial fibrosis $(37,38)$. The PI3K/AKT signal transduction pathway is closely associated with cell growth and proliferation. It regulates the development of renal diseases including renal fibrosis, diabetic nephropathy, autosomal dominant polycystic kidney disease and renal cell carcinoma, along with the level of p-AKT in the whole kidney following 4 weeks of diabetes in rats $(39,40)$.

In this study, amlodipine besylate combined with AA-TCM nursing in the treatment of renal failure hypertension, can effectively reduce the systolic pressure, diastolic pressure, and improve the symptoms of patients. Amlodipine besylate combined with AA-TCM nursing in the treatment of renal failure hypertension may be associated with the regulation of the expression of the PI3K/AKT pathway gene protein, so as to regulate the expression of BUN, CRE, UP, BUP and NAG.

The present study confirms that amlodipine combined with AA-TCM nursing can effectively treat renal failure hypertension; its antihypertensive effect is very good and the clinical symptoms have been improved. This result is associated with the influence of the PI3K/AKT pathway. The present study provides a clinical basis for the treatment of renal failure and hypertension.

\section{Acknowledgements}

Not applicable.

\section{Funding}

The present study was supported by the Traditional Chinese Medicine Science and Technology Development Program of Shandong Province (grant no. 2017-387).

\section{Availability of data and materials}

All data generated or analyzed during this study are included in this published article.

\section{Authors' contributions}

LF, RC and WL conceived and designed the experiments. LF, JS, RC, QZ, SL and WL performed the experiments, prepared the manuscript and analyzed the data. All authors read and approved the final version of the manuscript.

\section{Ethics approval and consent to participate}

The study was approved by the Yantai Traditional Chinese Medicine Hospital (Yantai, China). Informed consent was obtained from all participants. All included patients fulfilled criteria and completed the study.

\section{Patient consent for publication}

All participants provided written informed consent.

\section{Competing interests}

The authors declare that they have no competing interests.

\section{References}

1. Levey AS, Atkins R, Coresh J, Cohen EP, Collins AJ, Eckardt KU, Nahas ME, Jaber BL, Jadoul M, Levin A, et al: Chronic kidney disease as a global public health problem: Approaches and initiatives-a position statement from kidney disease improving global outcomes. Kidney Int 72: 247-259, 2007.

2. Zoccali C, Kramer A and Jager KJ: Chronic kidney disease and end-stage renal disease-a review produced to contribute to the report 'the status of health in the European union: Towards a healthier Europe'. NDT Plus 3: 213-224, 2010.

3. Brück K, Stel VS, Gambaro G, Hallan S, Völzke H, Ärnlöv J, Kastarinen M, Guessous I, Vinhas J, Stengel B, et al: CKD prevalence varies across the European general population. J Am Soc Nephrol 27: 2135-2147, 2016.

4. Symplicity HTN-2 Investigators, Esler MD, Krum H, Sobotka PA, Schlaich MP, Schmieder RE and Böhm M: Renal sympathetic denervation in patients with treatment-resistant hypertension (The Symplicity HTN-2 Trial): A randomised controlled trial. Lancet 376: 1903-1909, 2010.

5. Makani H, Bangalore S, Romero J, Wever-Pinzon $\mathrm{O}$ and Messerli FH: Effect of renin-angiotensin system blockade on calcium channel blocker associated peripheral edema. Am J Med 124: 128-135, 2011.

6. Dimitrow PP and Jawien M: Anti-inflammatory effect of atorvastatin in patients with aortic sclerosis or mild aortic stenosis independent of hypercholesterolemia. Pharmacol Rep 62: 1250-1254, 2010.

7. Benicky J, Sanchez-Lemus E, Honda M, Pang T, Orecna M, Wang J, Leng Y, Chuang DM and Saavedra JM: Angiotensin II AT1 receptor blockade ameliorates brain inflammation. Neuropsychopharmacology 36: 857-870, 2011.

8. Kim KH and Kim WS: Chronic unilateral chemosis following the use of amlodipine besylate. BMC Ophthalmol 14: 124, 2014.

9. Dai Y, Wang W, Sun Q and Tuohayi J: Ginsenoside Rg3 promotes the antitumor activity of gefitinib in lung cancer cell lines. Exp Ther Med 17: 953-959, 2019.

10. Chang XA, Dai S, Ye ZZ and Song QH: Treatment of facial acne vulgaris by chinese medicine combined western medicine. Zhongguo Zhong Xi Yi Jie He Za Zhi 37: 160-164, 2017 (In Chinese).

11. Su L, Meng L, Chen R, Wu W, Peng B and Man L: Acupoint application for asthma therapy in adults: A systematic review and meta-analysis of randomized controlled trials. Forsch Komplementmed 23: 16-21, 2016.

12. Liu CY, Qin S, Liu LY, He YM, Wang HS, Wu WZ, Chen D and Zhang C: Inhibitory effect of acupoint application on airway remodeling and expression of TGF- $\beta 1 /$ Smad 3 in the lung tissue of chronic asthma mice. Zhen Ci Yan Jiu 42: 153-158, 2017 (In Chinese).

13. Fresno Vara JA, Casado E, de Castro J, Cejas P, Belda-Iniesta C and González-Barón M: PI3K/AKT signalling pathway and cancer. Cancer Treat Rev 30: 193-204, 2004.

14. Xu X, Cao Z, Cao B, Li J, Guo L, Que L, Ha T, Chen Q, Li C and Li Y: Carbamylated erythropoietin protects the myocardium from acute ischemia/reperfusion injury through a PI3K/AKT-dependent mechanism. Surgery 146: 506-514, 2009.

15. Tramontano AF, Muniyappa R, Black AD, Blendea MC, Cohen I, Deng L, Sowers JR, Cutaia MV and El-Sherif N: Erythropoietin protects cardiac myocytes from hypoxia-induced apoptosis through an AKT-dependent pathway. Biochem Biophys Res Commun 308: 990-994, 2003.

16. Zhang J, Yao Y, Xiao F, Lan X, Yu C, Zhang Y, Jiang C, Yang J, Pei G, Li Y, et al: Administration of dexamethasone protects mice against ischemia/reperfusion induced renal injury by suppressing PI3K/AKT signaling. Int J Clin Exp Pathol 6: 2366-2375, 2013.

17. Xie L, Zheng X, Qin J, Chen Z, Jin Y and Ding W: Role of PI3-kinase/AKT signalling pathway in renal function and cell proliferation after renal ischaemia/reperfusion injury in mice. Nephrology (Carlton) 11: 207-212, 2006.

18. Matheny RW and Adam ML: Current perspectives on AKTAKT-ivation and AKT-ions. Exp Biol Med (Maywood) 234: 1264-1270, 2009. 
19. Liu L, Wu Z, Zhu D, et al: The guideline 2010 for hypertension prevention and treatment in China. 3rd edition, 2010. http:// csjfyy.com/articles/E/020/010/050.pdf.

20. National Research Council: The Guide for the Care and Use of Laboratory Animals. National Academies Press, Washington DC, revised 1996.

21. Livak KJ and Schmittgen TD: Analysis of relative gene expression data using real-time quantitative PCR and the 2(-Delta Delta C(T)) method. Methods 25: 402-408, 2001.

22. Bhatt DL, Kandzari DE, O'Neill WW, D'Agostino R, Flack JM, Katzen BT, Leon MB, Liu M, Mauri L, Negoita M, et al: A controlled trial of renal denervation for resistant hypertension. $\mathrm{N}$ Engl J Med 370: 1393-1401, 2014

23. Zoccali C, Tripepi R, Torino C, Tripepi G and Mallamaci F: Moderator's view: Ambulatory blood pressure monitoring and home blood pressure for the prognosis, diagnosis and treatment of hypertension in dialysis patients. Nephrol Dial Transplant 30: $1443-1448,2015$.

24. Azizi M, Sapoval M, Gosse P, Monge M, Bobrie G, Delsart P, Midulla M, Mounier-Véhier C, Courand PY, Lantelme P, et al: Optimum and stepped care standardised antihypertensive treatment with or without renal denervation for resistant hypertension (DENERHTN): A multicentre, open-label, randomised controlled trial. Lancet 385: 1957-1965, 2015

25. Agarwal R: Pro: Ambulatory blood pressure should be used in all patients on hemodialysis. Nephrol Dial Transplant 30: 1432-1437, 2015.

26. Jardine AG: Con: Ambulatory blood pressure measurement in patients receiving haemodialysis: A sore arm and a waste of time? Nephrol Dial Transplant 30: 1438-1441, 2015.

27. Bansal N, McCulloch CE, Rahman M, Kusek JW, Anderson AH Xie D, Townsend RR, Lora CM, Wright J, Go AS, et al: Blood pressure and risk of all-cause mortality in advanced chronic kidney disease and hemodialysis: The chronic renal insufficiency cohort study. Hypertension 65: 93-100, 2015.

28. Say EA, Shields CL, Bianciotto $\mathrm{C}$ and Shields JA: Chronic conjunctival chemosis from amlodipine besylate (Norvasc). Cornea 30: 604-607, 2011.

29. Pedrinelli R, Dell'Omo G and Mariani M: Calcium channel blockers, postural vasoconstriction and dependent oedema in essential hypertension. J Hum Hypertens 15: 455-461, 2001.

30. Moes AD, Hesselink DA, van den Meiracker AH, Zietse R and Hoorn EJ: Chlorthalidone versus amlodipine for hypertension in kidney transplant recipients treated with tacrolimus: A randomized crossover trial. Am J Kidney Dis 69: 796-804, 2017.
31. Houben AJ, Burgwinkel JP and de Leeuw PW: A novel approach to the study of human microcirculation: Reactivity to locally applied angiotensin II in the conjunctivalmicrovascular bed. J Hypertens 24: 2225-2230, 2006.

32. Weir MR, Rosenberger C and Fink JC: Pilot study to evaluate a water displacement technique to compare effects of diuretics and ACE inhibitors to alleviate lower extremity edema due to dihydropyridine calcium antagonists. Am J Hypertens 14: 963-968, 2001.

33. Hu Q, Gu P, Jiang X, Jiang F, Zhuang J, Zheng H, Yang G and Jiang S: Moderate and severe persistent allergic rhinitis treated with acupoint application therapy of the different intensity: A randomized controlled trial. Zhongguo Zhen Jiu 37: 1177-1182, 2017 (In Chinese).

34. Ooms LM, Fedele CG, Astle MV, Ivetac I, Cheung V, Pearson RB, Layton MJ, Forrai A, Nandurkar HH and Mitchell CA: The inositol polyphosphate 5-phosphatase, PIPP, is a novel regulator of phosphoinositide 3-kinase-dependent neurite elongation. Mol Biol Cell 17: 607-622, 2006.

35. Jean $\mathrm{S}$ and Kiger AA: Classes of phosphoinositide 3-kinases at a glance. J Cell Sci 127: 923-928, 2014.

36. Scheid MP, Huber M, Damen JE, Hughes M, Kang V, Neilsen P, Prestwich GD, Krystal G and Duronio V: Phosphatidylinositol $(3,4,5) \mathrm{P} 3$ is essential but not sufficient for protein kinase $\mathrm{B}$ (PKB) activation phosphatidylinositol $(3,4) \mathrm{P} 2$ is required for PKB phosphorylation at Ser-473: Studies using cells from SH2-containing inositol5-phosphatase knockout mice.J Biol Chem 277: 9027-9035, 2002.

37. Ma K, Cheung SM, Marshall AJ and Duronio V: PI $(3,4,5) \mathrm{P} 3$ and $\mathrm{PI}(3,4) \mathrm{P} 2$ levels correlate with $\mathrm{PKB} /$ akt phosphorylation at Thr308 and Ser473, respectively PI(3,4)P2 levels determine PKB activity. Cell Signal 20: 684-694, 2008.

38. Li H and Marshall AJ: Phosphatidylinositol $(3,4)$ bisphosphate-specific phosphatases and effector proteins: A distinct branch of PI3K signaling. Cell Signal 27: 1789-1798, 2015.

39. Inoki K, Li Y,Zhu T, Wu J and Guan KL: TSC2 is phosphorylated and inhibited by AKT and suppresses mTOR signalling. Nat Cell Biol 4: 648-657, 2002.

40. Wang $\mathrm{F}$, Ijuin $\mathrm{T}$, Itoh $\mathrm{T}$ and Takenawa $\mathrm{T}$ : Regulation of IGF-1/PI3K/AKT signaling by the phosphoinositide phosphatase pharbin. J Biochem 150: 83-93, 2011. 